\section{MS12-P13 How multivalent salts help proteins crystallize}

Fajun Zhang ${ }^{1}$, Georg Zocher ${ }^{2}$, Thilo Stehle ${ }^{2,3}$, Frank Schreiber ${ }^{1}$

1. Institut für Angewandt Physik, Universität Tübingen

2. IFIB, University of Tübingen

3. Department of Pediatrics, Vanderbilt University School of Medicine, Nashville, USA

email: fajun.zhang@uni-tuebingen.de

Knowledge of the protein structure is considered a precondition for understanding their function. Crystallography remains the most powerful tool in that context, but crystal structure determination of macromolecules is often hampered by a lack of crystals suitable for diffraction. In our work, we have studied the crystallization of human serum albumin (HSA) and bovine beta-lactoglobulin (BLG) in the presence of yttrium chloride (and other multivalent salts) to yield high quality crystals with new crystal packing. We demonstrate that trivalent salts can be used to optimize crystallization conditions for globular proteins along general physical insights. We have revealed that negatively charged proteins at neutral $\mathrm{pH}$ in the presence of trivalent salts undergo a "reentrant condensation" phase behavior [1-3], i.e. a phase-separated regime occurs in between two critical salt concentrations, $\mathrm{c}^{*}<\mathrm{c}^{* *}$, giving rise to a metastable liquid-liquid phase separation (LLPS) $[3,4]$. Crystallization from the condensed regime follows different mechanisms. Near $\mathrm{c}^{*}$, crystals grow following a classic nucleation and growth mechanism; near LLPS binodal, crystallization follows a two-step mechanism, i.e, crystals growth follows a metastable LLPS [4-8]. The yttrium ions are not only used to engineer crystallization, but are an integral part of the crystal lattice and can therefore be used to solve the phase problem using anomalous dispersion methods. The structure analysis demonstrates the specific binding of yttrium ions to surface-exposed glutamate and aspartate side chains contributed by different molecules in the crystal lattice. By bridging molecules in this manner, contacts between molecules are formed that enable the formation of a stable crystal lattice. Based on this bridging effect, an ion-activated patchy colloidal model was proposed to rationalize the phase behaviour observed experimentally [9].

References [1] F. Zhang et al., Phys. Rev. Lett. 2008, 101, 148101 [2] F. Zhang et al., Proteins 2010, 78, 3450 [3] F. Zhang et al., Soft Matter 2012, 8, 1313 [4] F. Zhang et al., J. Appl. Cryst. 2011, 44, 755 [5] F. Zhang et al., Faraday Discuss. 2012, 159, 313 [6] F. Roosen-Runge et al., J. Phys. Chem.B 2013, 117, 5777 [7] A. Sauter, et al. JACS, 2015, 137, 1485 [8] A. Sauter, et al. Faraday Discuss. 179, 41 [9] F. Roosen-Runge et al. Scientific Reports, 2014, 4, 7016.

Keywords: protein crystallization, nonclassical nucleation, liquid-liquid phase separation, multivalent salt

\section{MS12-P14 Structural and ligand-binding properties of fatty acid-binding proteins (pFABP4 and pFABP5) in the gentoo penguin \\ Chang woo Lee ${ }^{1,2}$, Jung Eun Lee ${ }^{2}$, Hyun Park ${ }^{1,2}$, Il-Chan Kim², Jun Hyuck Lee e $^{1,2}$}

1. Department of Polar Sciences, University of Science and Technology, Incheon 406-840, Republic of Korea

2. Division of Polar Life Sciences, Korea Polar Research Institute, Incheon 406-840, Republic of Korea

email: justay@kopri.re.kr

Fatty acid-binding proteins (FABPs) are small, widely expressed intracellular proteins located in various tissues. FABPs facilitate the solubilization and transport of fatty acids and other hydrophobic substances by directly interacting with ligands. Here, we report the crystal structures of ligand-unbound pFABP4, linoleate-bound pFABP4, and palmitate-bound pFABP5 from the gentoo penguin (Pygoscelis papua) at 2.1, 2.2, and $2.3 \AA$ resolution, respectively. The pFABP4 and pFABP5 structures were comprised primarily of 10-stranded $\beta$-barrels and two short $\alpha$-helices forming a cap region. Inside the $\beta$-barrel, a hydrophobic cavity containing the ligand binding site was observed. A ligand binding test using 8-anilinonaphthalene-1-sulfonic acid indicated that pFABP4 has relatively high affinity for linoleate compared with pFABP5. In contrast, pFABP5 has high affinity for palmitate compared with pFABP4. Structure comparison revealed the largest difference in the $\beta 3-\beta 4$ loop region. Upon palmitate binding to pFABP5, the $\beta 3-\beta 4$ loop was moved by $4 \AA$ compared to linolate-bound pFABP4. Moreover, several sequences (A76/L78, T30/M32, underlining indicates pFABP4 residues) caused structural changes and different affinities for linoleate and palmitate. Structural comparisons, combined with a ligand-binding assay, provide insight into the mechanism of the ligand specificities of pFABP4 and pFABP5.

Keywords: Fatty acid-binding protein, $\beta$-barrel protein, crystal structure, Gentoo penguin (Pygoscelis papua), X-ray crystallography 